\title{
Signatures of the electromagnetic field quantization in the nonlinear optical response of excitons
}

\author{
Salvatore Savasta and Raffaello Girlanda \\ INFM and Dipartimento di Fisica della Materia e Tecnologie Fisiche Avanzate, Università di \\ Messina, Salita Sperone 31, I-98166 Messina, Italy \\ E-mail: girlanda@imeuniv.unime.it
}

\begin{abstract}
We present a fully quantum mechanical theory for an interacting system of photons and Coulomb correlated electrons and holes in a semiconductor, using a pertubation series in the exciting laser field. The theory provides microscopic descriptions of nonlinear optical processes in semiconductors related to the electromagnetic field quantization. As an application of the theory we show that it is possible to transfer the exciton-exciton Coulomb correlation to photons, thus producing pairs of near-gap photons with a high degree of quantum entanglement. The photon pairs emerge from the spontaneous optical decay of biexcitons into two polaritons. The pair intensity correlations, calculated in the low density limit for a $\mathrm{CuCl}$ slab and for semiconductor microcavities, exhibit quantum features which can be observed by coincidence detection.
\end{abstract}

\section{Introduction}

In studying the optical processes in semiconductor systems, much can be learned by regarding the semiconductor system as an ensemble of noninteracting, two level atoms interacting with a classical radiation field. The dynamics of such a system is governed by the well known optical Bloch equations $[1,2]$. However the proximity of the atoms in a solid and the strong Coulomb interaction between the electrons in semiconductors give rise to phenomena that cannot be described by the optical Bloch equations. The pertinent theory describing the dynamics of the semiconductor electrons under the influence of the driving laser field is based on a generalization of the optical Bloch equations including the Coulomb correlation between electrons, namely the semiconductor Bloch equations (SBE) [3]. SBE without any approximation have the structure of an open hierarchy of equations. Whenever one is dealing with a hierarchy of dynamical objects, one is faced with the problem of finding an appropriate termination procedure. One self-consistent approximation scheme leads to the Hartree-Fock semiconductor Bloch equations (HFSBE) [3-6]. In HFSBE the termination is obtained by factorizing the many body correlations using single-particle densities. The factorization implies a limitation of the Coulomb correlation at first order. Results obtained within HFSBE agreed well with the experimental findings in a wide range of semiconductor bulk and confined systems. These effective equations provide a physical picture of the origin of nonlinearities in the optical response of semiconductor systems. As shown first by Combescot and Combescot [7], despite the wide range of applicability, HFSBE are not able to describe those effects originating from states with two electron-hole pairs. Effects such as the polarization-dependent response of excitons, the red-shift Stark effect of excitons, 
the bound biexciton signatures in two photon absorption and in photoluminescence emission, cannot be described by HFSBE and lead to more refined theoretical investigations beyond the HFSBE.

The inclusion of four particle correlations in the optical response of the semiconductor has been achieved by introducing a different truncation scheme. Axt and Stahl [8] in the so-called dynamics controlled truncation scheme (DCTS), based the truncation scheme on the smallness of the optical excitation. Axt and Stahl showed that once the perturbative order in the excitation strength has been fixed, the dynamics of the interacting electron system can be described by a closed set of equations. Within this approach, once the perturbative order in the excitation strength has been fixed, the Coulomb correlation contributing to the optical response up to fixed order is included exactly. By applying the DCTS, it can be shown that the third order nonlinear reponse depends only on one $e h$-pair subspace (excitons) and the two $e h$-pair subspace (biexcitons) [9], hence the third order nonlinear response is intimately tied to the dynamics of two and four-particle (two electrons and two holes) states. Östreich et al [10], provided an exact numerical evaluation of the four particle correlation determining the third order nonlinear response.

SBE and the corresponding approximation schemes outlined above describe the dynamics of semiconductor electrons interacting with a classical light field. The vast majority of optical processes in bulk and in confined semiconductor systems can be adequately explained using the classical theory of electromagnetic radiation. However, although the investigations of the optical properties of semiconductors have been developed independently of quantum optics, it is well known that light field quantization is an essential ingredient for a proper description of semiconductor optics. The simplest example of the importance of field quantization in semiconductors is the spontaneous radiative recombination of an electron and a hole, which is the underlying process of light generation in the light-emitting diode and in photoluminescence experiments [11]. Although spontaneous radiative recombination can be included phenomenologically in semiclassical treatments, it is a process tied to electromagnetic field quantization.

Another important example is provided by the hyper Raman scattering (HRS) process. HRS is known as an efficient technique of nonlinear optical spectroscopy applied to the investigation of bulk polariton dispersions [12]. HRS can be schematically described as follows: two incident photons of given energy and wave vectors propagate inside the crystal as polaritons and create a virtual state with two electron-hole pairs which acts as intermediate state to create two final polaritons or a longitudinal exciton and a polariton. Energy and momentum are conserved in the whole process. The emission line from one final polariton can be detected experimentally. The determination of the polariton dispersion has successfully been accomplished by HRS in several large-gap bulk semiconductors [12]. HRS is a process related to third-order nonlinear susceptibility, and can be considered as spontaneous nondegenerate four-wave mixing (FWM). In FWM the optical decay of the virtually excited biexcitons is stimulated by sending an additional light beam, while in HRS the decay is determined by intrinsic vacuum fluctuations. Nonlinear spontaneous processes occupy a central position in the field of quantum optics, and are well known to be sources of nonclassical light [13]. HRS, being a nonlinear spontaneous process where many-body correlations and vacuum fluctuations come together, represents an important and unexplored bridge between semiconductor nonlinear-optics and quantum optics. The microscopic description of HRS poses a considerable challenge to current theories since it requires a full quantum analysis of the semiconductor nonlinear response beyond the HFSBE which are not able to include four particle correlations and hence effects from states with two electron-hole pairs.

We have extended the DCTS, to include the electromagnetic field quantization [14-16]. In 
this way we were able to include the Coulomb correlation beyond first order as well as the field vacuum fluctuations. In this paper we present a microscopic theory of exciton interaction effects in nonlinear optical processes. Furthermore we analyse the signatures of the electromagnetic field quantization in the nonlinear optical response of excitons. In particular in section 5, by applying the extended DCTS, we show that the nonlinearly correlated excitations in bulk semiconductors and in semiconductor microcavities can produce entangled near-gap photon pairs which can be observed by coincidence detection.

Entangled particles have been produced in a variety of systems. Photon pairs produced by parametric down conversion [17] is the most known example. Entanglement describes a composite quantum system that cannot be factored into a product of single-particle states, and therefore has no classical counterpart. It is manifested by the potential to exhibit correlations that cannot be obtained with classical systems [18]. The theory of quantum information processing lies in entanglement. Teleportation of quantum states [19,20], quantum computation steps [21] and quantum cryptography [22] are recent striking applications of entanglement. Owing to the possibility of engineering the valence and conduction electronic states of semiconductors opened up by modern growth techniques, and owing to the possibility of controlling the exciton-photon interaction in semiconductor microcavities (MCs) [23], the generation of entangled photon pairs in semiconductor systems is expected to be promising in realizing integrated quantum-optical devices.

\section{The exciton-photon system}

We consider the problem of a semiconductor coupled to a photon field by electron polarization. We start from the Hamiltonian $\hat{H}_{s}=\hat{H}_{0}+\hat{V}_{\text {Coul }}$ of the usual semiconductor model [3,7], which is composed of a free-particle part $\hat{H}_{0}$ and the Coulomb interaction $\hat{V}_{\text {Coul }}$. We consider a zinc blende like semiconductor band structure. The valence band is made from p-like $(l=1)$ orbital states which, after spin-orbit coupling, give rise to $j=3 / 2$ and $j=1 / 2$ decoupled states. In materials like $\mathrm{CuCl}$, the upper valence band is twofold degenerate $(j=1 / 2)$, while in materials like GaAs it is fourfold degenerate $(j=3 / 2)$. The conduction band, arising from an s-like orbital state $(l=0)$, gives rise to $j=1 / 2$ twofold states. In the following, for the sake of simplicity, we will refer to materials like bulk $\mathrm{CuCl}$ and will only consider states from the twofold upper valence band and lower conduction band. Analogous results can be obtained by considering the fourfold valence band and considering quantum structures as quantum wells (QWs). Only $e h$ pairs with total angular momentum $J=1$ are dipole active in optical interband transitions. Photons with circular polarizations $\sigma=+(-)$ excite $e$ with $m_{e}=+\left(m_{e}=-\right)$ and $h$ with $m_{h}=+\left(m_{h}=-\right)$.

An important feature of $\hat{H}_{s}$ is that its matrix elements between states with a different number of $e h$ pairs are zero. As a consequence it does not couples states with different numbers of $e h$ pairs.

The eigenstates $|N, \alpha, \boldsymbol{k}\rangle$ with energy $\omega_{N \alpha \boldsymbol{k}}$ of $\hat{H}_{S}$ can be labelled according to the number $N$ of $e h$ pairs and the total momentum $k[7,10]$. The state with $N=0$ is the semiconductor ground state and corresponds to the full valence band. The $N=1$ subspace is the exciton subspace. Optically active exciton states can be labelled with the additional quantum number $\alpha=(n, \sigma)$ where $\sigma$ indicates the spin (e.g. $\sigma=+$ indicates an $e h$ pair with $m_{e}=+$ and $\left.m_{h}=+\right)$ and $n$ spans all the bound and unbound exciton levels. These states can be obtained by applying the exciton creation operator

$$
\hat{B}_{n, \sigma, k}^{\dagger}=\sum_{k^{\prime}} \Phi_{n, \sigma, k^{\prime}}^{*} \hat{c}_{\sigma, k^{\prime}+k / 2}^{\dagger} \hat{d}_{\sigma,-k^{\prime}+k / 2}^{\dagger}
$$

to the semiconductor ground state, where the exciton wave function $\Phi_{n, \sigma k^{\prime}}$ is the solution to the 
Wannier equation for the semiconductor. The set of bound and unbound states with $N=2 \mathrm{eh}$ pairs, determining the biexciton subspace, can be obtained solving the corresponding secular equation [24], or by using a simpler interaction model [25].

By denoting as $\mu_{\sigma}$ the interband dipole matrix element between the Bloch states of the bands, the $\sigma$ electronic polarization density at wave vector $k$ is given by

$$
\hat{P}_{\sigma, k}^{(+)}=\mu_{\sigma}^{*} \sum_{k^{\prime}} \hat{c}_{\sigma, k^{\prime}+k / 2} \hat{d}_{\sigma,-k^{\prime}+k / 2}
$$

and can be expressed in terms of exciton operators

$$
\hat{P}_{\sigma, k}^{(+)}=\mu_{\sigma}^{*} \sum_{n, \boldsymbol{k}^{\prime}} \Phi_{n, \sigma, k^{\prime}}^{*} \hat{B}_{n, \sigma, k}=\sum_{n} M_{n \sigma}^{*} \hat{B}_{n, \sigma, k} \equiv \sum_{n} \hat{P}_{n, \sigma, k}^{(+)}
$$

The total $\sigma$ electronic polarization at wave vector $k$ is $\hat{P}_{\sigma k}=\hat{P}_{\sigma k}^{(+)}+\hat{P}_{\sigma k}^{(-)}, \hat{P}^{(-)}$being the Hermitian conjugate of $\hat{P}^{(+)}$.

In the following, in order to analyse the dynamical evolution of the electron polarization, we will use the projection operators defined by

$$
\hat{X}_{N, \alpha, \boldsymbol{k} ; N^{\prime}, \alpha^{\prime}, \boldsymbol{k}^{\prime}}=|N, \alpha, \boldsymbol{k}\rangle\left\langle N^{\prime}, \alpha^{\prime}, \boldsymbol{k}^{\prime}\right| .
$$

By using the completeness relation $\mathbf{1} \equiv \sum_{N, \alpha, k} \hat{X}_{N, \alpha, k ; N, \alpha, k}$, the exciton operators can be expanded in terms of projection operators [10]

$$
\hat{B}_{n, \sigma, k}=\hat{X}_{0 ; 1, n, \sigma, k}+\sum_{N \geqslant 1, \alpha^{\prime}, \alpha^{\prime \prime}, \boldsymbol{k}^{\prime} \boldsymbol{k}^{\prime \prime}}\left\langle N, \alpha^{\prime}, \boldsymbol{k}^{\prime}\left|\hat{\boldsymbol{B}}_{n, \sigma, \boldsymbol{k}}\right| N+1, \alpha^{\prime \prime}, \boldsymbol{k}^{\prime \prime}\right| \hat{X}_{N, \alpha^{\prime}, \boldsymbol{k}^{\prime} ; N+1, \alpha^{\prime \prime}, \boldsymbol{k}^{\prime \prime}}
$$

The translation symmetry obviously implies $\boldsymbol{k}^{\prime \prime}-\boldsymbol{k}^{\prime}=\boldsymbol{k}$. Before going on, we point out that $\alpha$, together with the number $N$ of $e h$ pairs and with the wave vector $k$, spans the eigenstates of Hamiltonian $\hat{H}_{s}$. When referred to exciton states $(N=1)$, or to exciton operators $\hat{B}_{\alpha, k}^{\dagger}$, $\alpha \equiv(n, \sigma)$.

The interaction of the electrons of the semiconductor with a photon field is given in the usual dipole and rotating wave approximations

$$
\hat{H}_{I}=-\sum_{\sigma, k} \hat{P}_{\sigma k}^{(-)} \hat{E}_{\sigma k}^{(+)}+\text {H.c. }
$$

where $\hat{E}_{\sigma k}^{(+)}=(1 / \mathcal{V}) \int \mathrm{d}^{3} r \hat{E}_{\sigma}^{(+)}(r) \mathrm{e}^{-\mathrm{i} k \cdot r}$ is the component of the positive part of the electricfield operator with wave vector $k$ and $\mathcal{V}$ the quantization volume. The total electric-field operator is $\hat{E}(\boldsymbol{r})=\sum_{\sigma}\left(\hat{E}_{\sigma}^{(+)}(\boldsymbol{r})+\hat{E}_{\sigma}^{(-)}(\boldsymbol{r})\right)$ and $\hat{E}_{\sigma}^{(-)}(\boldsymbol{r})$ the Hermitian conjugate of $\hat{E}_{\sigma}^{+}(\boldsymbol{r})$.

Let us now consider the electromagnetic field quantization in the absence of the semiconductor system. We quantize the field propagating inside a general dielectric planar and nonabsorbing system. For example, the system can be either a planar dielectric microcavity in the absence of the active semiconductor medium (e.g. the QWs), or a bulk slab of given background dielectric constant $\varepsilon_{b}$. By exploiting the planar symmetry of the dielectric system we can assume translational symmetry in the incidence plane so that the in-plane component $\boldsymbol{p}=\left(k_{x}, k_{y}, 0\right)$ of the wave vector $\boldsymbol{k}$ is a good quantum number for the whole system. In the multipolar form of the interaction adopted here, the field coordinate is given by the vector potential, while the conjugate momentum is given by the displacement operator. By using the usual quantization scheme for the transverse electromagnetic field, we can expand the field variables in terms of the photon operators $\hat{a}_{\sigma, p}(\omega)$. The photon Hamiltonian in the absence of the interacting semiconductor system, in terms of photon operators reads

$$
H_{p h}=\sum_{\sigma, \boldsymbol{p}, \omega} \omega_{\sigma, \boldsymbol{p}}(\omega) \hat{a}_{\sigma, \boldsymbol{p}}^{\dagger}(\omega) \hat{a}_{\sigma, \boldsymbol{p}}(\omega) .
$$


The expansion of the operators of the electronic system in terms of projection operators is useful for introducing the DCTS [10]. As we have pointed out in the introduction, and as it is known from semiconductor nonlinear optics $[9,26]$, the theory describing the dynamics of the semiconductor electrons interacting with a light field has the structure of an open hierarchy of dynamical variables. Whenever one is dealing with an open hierarchy of dynamical objects, one is facing the problem of finding an appropriate termination procedure. Here we present an extension of the truncation procedure introduced by Axt and Stahl [9] to include the quantization of the light field and polariton effects. Such a truncation scheme is analogous to the classification scheme of nonlinear optical processes, where the coherent input light beam represents the expansion parameter. Here we label the photon modes simply by $k$. For the sake of simplicity we shall consider a single mode coherent input electric field, $\left\langle\hat{a}_{k_{I}}\left(t_{0}\right)\right\rangle=\mathcal{E}$ being the expectation value of its photon operator. Generalization to a multimode input field is straightforward. We consider the following operators acting on the electron-photon system

$$
\hat{X}_{N, \alpha, k ; N^{\prime}, \alpha^{\prime}, k^{\prime}} \prod_{k, k^{\prime}} \hat{a}_{k}^{\dagger n_{k}} \hat{a}_{k^{\prime}}^{n_{k^{\prime}}^{\prime}} .
$$

Operators defined in equation (6) govern the dynamics of the photon-electron interacting system. The truncation scheme is based on the expansion of the expectation values of these operators in terms of power series in the input field. We have proved [16] the following theorem.

Theorem. The expectation value of operators of the electron-photon system defined in equation (6), subjected to Hamiltonians $\hat{H}_{s}, \hat{H}_{p h}$ and $\hat{H}_{I}$, can be expressed in terms of a power series in the input field as

$$
\begin{gathered}
\left\langle\hat{X}_{N, \alpha, k ; N^{\prime}, \alpha^{\prime}, k^{\prime}} \prod_{k, k^{\prime}} \hat{a}_{k}^{\dagger n_{k}}, \hat{a}_{k^{\prime}}^{n_{k^{\prime}}^{\prime}}\right\rangle=\sum_{i=0}^{i_{0}}\left\langle\hat{X}_{N, \alpha, k ; N^{\prime}, \alpha^{\prime}, k^{\prime}} \prod_{k, k^{\prime}} \hat{a}_{k}^{\dagger n_{k}}, \hat{a}_{k^{\prime}}^{n_{k^{\prime}}^{\prime}}\right\rangle^{\left(N+N^{\prime}+n+n^{\prime}+2 i_{0}\right)} \\
+\mathcal{O}\left(\mathcal{E}^{N+N^{\prime}+n+n^{\prime}+2 i_{0}+2}\right)
\end{gathered}
$$

where $n=\sum_{k} n_{k}$ and the superscripts indicate the power order in $\mathcal{E}$ and $\mathcal{O}\left(\mathcal{E}^{j}\right)$ indicates the terms of order $\geqslant j$.

The theorem permits a truncation of the hierarchy of equations for the expectation values of operators defined in equation (6). It can be shown that the equation of motion for a given operator with $\bar{p}=N+N^{\prime}+n+n^{\prime}$ introduces an open hierarchy of equations for operators with $p=\bar{p}+2 i_{0}$ with $i_{0}>0$. Once a perturbative order $p_{M}$ has been fixed, the theorem establishes that the hierarchy of equations for expectation values is closed, and only operators with $p \leqslant p_{M}$ are to be considered. By inspecting the Heisenberg equation of motion for the electron-photon system and applying the theorem, one finds that to calculate field intensities at the lowest nonlinear order, only the dynamics of electronic states with $N=1$ and $N=2$ has to be included. Analogous considerations and inspections can be brought on when considering perturbative calculations of higher order field-field correlation functions. As a last remark we point out that the validity of the theorem can be extended to include the interaction of the electron system with a phonon thermal bath.

\section{The equations of motion for the exciton-photon system}

In order to calculate the dynamical evolution of the electronic polarization in a photon field, we consider the Heisenberg equation of motion for the exciton operators $\hat{B}_{\alpha, k}$. For the sake of simplicity we drop the labels describing wave vectors and polarizations. By expanding 
the exciton operators in terms of projection operators (3) and using the fact that projection operators are built on from eigenstates of $H_{s}$ we obtain

$$
i \frac{\partial}{\partial t} \hat{B}_{n}=\omega_{1, n} \hat{B}_{n}-M_{\alpha} \hat{E}^{+}+\sum_{N \geqslant 1, \alpha^{\prime}} \hat{X}_{0 ; N, \alpha^{\prime}}^{\dagger} \hat{R}_{\alpha ; N, \alpha^{\prime}}^{(N+1)}
$$

where we have defined the correlation force

$$
\hat{R}_{\alpha ; N, \alpha^{\prime}}^{(N+1)}=\sum_{\alpha^{\prime \prime}} c_{\alpha, \alpha^{\prime}, \alpha^{\prime \prime}}^{(N)} \hat{X}_{0 ; N+1, \alpha^{\prime \prime}}+\sum_{\alpha^{\prime \prime}} \Omega_{\alpha, \alpha^{\prime}, \alpha^{\prime \prime}}^{(N)} \hat{X}_{0 ; N, \alpha^{\prime \prime}} \hat{E}^{+} .
$$

The coefficients $\Omega^{(N)}$ and $c^{(N)}$ coincide with those obtained in the semiclassical theory of the nonlinear response in interacting electron system [10] and are given by

$$
\begin{aligned}
& c_{\alpha \alpha^{\prime} \alpha^{\prime \prime}}^{(N)}=\left(\omega_{N+1, \alpha^{\prime \prime}}-\omega_{N, \alpha^{\prime}}-\omega_{1, \alpha}\right)\left\langle N, \alpha^{\prime}\left|\hat{B}_{\alpha}\right| N+1, \alpha^{\prime \prime}\right\rangle \\
& \Omega_{n \sigma, \alpha^{\prime}, \alpha^{\prime \prime}}^{(N)}=\sum_{n_{2}} M_{n_{2}}\left\langle N, \alpha^{\prime}, \boldsymbol{k}^{\prime}\left|\left[\hat{B}_{n}, \hat{B}_{n_{2}}^{\dagger}\right]-\delta_{n, n 2}\right| N, \alpha^{\prime \prime}\right\rangle .
\end{aligned}
$$

The last term of equation (8) is the nonlinear source term for the interband polarization. Nonlinear optical effects [10] as well as quantum optical correlations [14] originate from $\hat{R}^{(N)}$. The first term in the r.h.s. of equation (9) comes from Coulomb interactions between electrons whereas the second term expresses the phase space filling and is directly related, as can be observed by looking at equation $(10 b)$, to the deviations of commutation relations of exciton operators from ideal bosonic rules. Replacing the electric-field operator by a classical field amplitude, and taking the expectation value, the operator equation (8) reduces to the corresponding semiclassical equation describing the exact nonlinear response of the semiconductor with Hamiltonian $\hat{H}_{s}$ and interacting with light [10].

The nonlinear source term in equation (8) is expressed as a summation of projection operators whose dynamics introduces a hierarchy of equations. The truncation of the hierarchy of equations can be achieved using a classification according to powers in the driving field as shown in section 2. In principle, the truncation theorem cannot be applied directly to operators; it applies to expectation values. However working with Heisenberg equations for operators is more convenient, and allows us to obtain more compact expressions. Once the perturbative order has been chosen, by inspecting the expectation values of interest (which are essentially the expectation values of the electric-field operator and of field-field correlations), one can see what the relevant operators are, whose dynamics has to be taken into account. In particular, in order to describe the lowest nonlinear order dynamics, it is sufficient to determine the lowest order nonlinear source term. In particular we have to write down the Heisenberg equation of motion for the operators $\hat{X}_{0 ; 2, \alpha}^{(2)}$ and $\hat{X}_{0 ; 1, \alpha}^{(1)}$, where the superscripts label the perturbative order in the input field of the corresponding expectation values. The projection operators in the truncated nonlinear source term obey the following equations

$$
\begin{aligned}
& i \frac{\partial}{\partial t} \hat{X}_{0 ; 1, n}^{(1)}=\omega_{1, n} \hat{X}_{0 ; 1, n}^{(1)}-M_{n} \hat{E}^{(1)+} \\
& i \frac{\partial}{\partial t} \hat{X}_{0 ; 2, \alpha}^{(2) \dagger}=\omega_{2, \alpha} \hat{X}_{0 ; 2, \alpha}^{(2) \dagger}-\sum_{n^{\prime} ; n^{\prime \prime}} M_{n^{\prime \prime}}\left\langle 2, \alpha\left|\hat{B}_{n^{\prime \prime}}\right| 1, n^{\prime}\right\rangle \hat{X}_{0 ; 1, n^{\prime}}^{(1)} \hat{E}^{(1)+} .
\end{aligned}
$$

Until now we have analysed the dynamics of the electronic polarization. In order to include properly the polariton effects we have to analyse the equations of motion for the light field. In particular, to obtain the lowest order nonlinear response, we have to derive an equation of motion for $\hat{E}^{(1)+}$ and $\hat{E}^{(3)+}$. From the Heisenberg equation of motion for the field variables, in 
the frequency space we can obtain the following wave equation for the electric field operator in an arbitrary planar dielectric system

$$
\left(\frac{\partial^{2}}{\partial z^{2}}+\varepsilon_{b}(z) \frac{\omega^{2}}{c^{2}}-p^{2}\right) \hat{E}_{p}^{+}(\omega, z)=-\frac{\omega^{2}}{\varepsilon_{0} c^{2}} \hat{P}_{p}^{+}(\omega, z)
$$

where $\varepsilon_{b}(z)$ is the background dielectric constant, and $\hat{P}_{p}^{+}(\omega, z)$ is the electronic polarization. For a bulk slab it is given by $P_{p}^{+}(\omega, z)=\sum_{k_{z}} \mathrm{e}^{\mathrm{i} k_{z} z} P_{\left(p, k_{z}\right)}^{+}(\omega)$ and, as we have seen in equation (1), can be expressed in terms of exciton operators. $\hat{P}_{p}^{+}(\omega, z)$ is the only source of nonlinearity. When analysing linear polariton propagation it is sufficient to include in equation (13) only the term

$$
\hat{P}_{p}^{(1)+}(\omega, z)=\sum_{n} M_{n}^{*} \sum_{k_{z}} \mathrm{e}^{\mathrm{i} k_{z} z} \hat{X}_{0 ; 1, n,\left(p, k_{z}\right)}^{(1)}(\omega) \equiv \sum_{n} M_{n}^{*} \hat{X}_{1, n, p}^{(1)}(\omega, z) .
$$

When analysing the lowest order nonlinear response, one also has to include in equation (13) the polarization term $\hat{P}_{p}^{(3)+}(\omega, z)$. The lowest order nonlinear quantum dynamics can thus be described by two coupled equations for $\hat{E}_{p}^{(3)+}$, and $\hat{P}_{p}^{(3)+}$ with $\hat{P}_{p}^{(3)+}$ driven by a nonlinear source term, including exciton and biexciton dynamics.

\section{Hyper Raman scattering}

Here we analyse the coherent nonlinear response up to the lowest non-zero order in the coherent input light field. In particular we analyse FWM and HRS emission at energies close to the semiconductor band edge. In the following we include explicitly the in-plane wave vector components $\boldsymbol{p}$ of the field and the electronic variables, while we omit polarization indices. The part of the source term in the equation of motion for $\hat{P}_{n, p}^{(3)+}(\omega, z)$ describing nonlinear processes induced by the Coulomb interaction is given by

$$
\hat{S}_{n, \sigma, p}^{(3)}(\omega, z)=\sum_{\alpha^{\prime}, p^{\prime}, p^{\prime \prime}} \int \mathrm{d} \omega^{\prime} \hat{X}_{1, \alpha^{\prime}, p^{\prime}}^{(1) \dagger}\left(\omega^{\prime}-\omega, z\right) \sum_{\alpha^{\prime \prime}} c_{\alpha \alpha^{\prime} \alpha^{\prime \prime}}^{(2)} \hat{X}_{2 \alpha^{\prime \prime}, p^{\prime \prime}}^{(2)}\left(\omega^{\prime}, z\right)
$$

where we have defined $\hat{X}_{2 \alpha, p}^{(2)}(\omega, z) \equiv \sum_{k_{z}} \mathrm{e}^{\mathrm{i} k_{z} z} \hat{X}_{0 ; 2, \alpha,\left(p, k_{z}\right)}^{(2)}(\omega)$. We point out that equation (14) has the same structure of the corresponding source term of the semiclassical theory (equation (6) of [10]), determining FWM via biexcitons. FWM can be schematically described as a two step semiclassical process: first a one pump beam of frequency $\omega_{I}$ and of given direction, creates a virtual biexciton grating $\left\langle\hat{X}_{2 \alpha, 2 p_{I}}\left(2 \omega_{I}, z\right)\right\rangle$, then the optical decay of the virtually excited biexcitons is stimulated by sending an additional probe light beam at $\omega^{\prime}$ which drives the exciton amplitude $\left\langle\hat{X}_{1, n, p^{\prime}}\left(\omega^{\prime}, z\right)\right\rangle$, given, according to equation (11) by

$$
\left\langle\hat{X}_{n, p^{\prime}}^{(1)}(\omega, z)\right\rangle=\chi_{n}^{(1)} \mathrm{e}^{\mathrm{i} k_{z}^{\prime} z} E_{p^{\prime}}\left(\omega^{\prime}\right) \delta\left(\omega-\omega^{\prime}\right)
$$

where $\chi_{n}^{(1)}=M_{n} /\left(\omega_{1, n}-\omega\right)$ and $E_{p^{\prime}}\left(\omega^{\prime}\right)$ is the amplitude of the coherent input probe field at frequency $\omega^{\prime}$. The source term for FWM emission at energy $\omega=2 \omega_{I}-\omega^{\prime}$ and in-plane wave vector $\boldsymbol{p}=2 \boldsymbol{p}_{I}-\boldsymbol{p}^{\prime}$ is thus determined by [10]

$$
\left\langle\hat{X}_{1, n, p^{\prime}}^{(1)}\left(\omega^{\prime}, z\right)\right\rangle^{*}\left\langle\hat{X}_{2 \alpha, 2 p_{I}}^{(2)}\left(2 \omega_{I}, z\right)\right\rangle
$$

In HRS the first step, i.e. the excitation of biexcitons by a one (or two) pump light beam, coincides with the first step in FWM, except the second step is different; in HRS there is no additional light beam and $\left\langle\hat{X}_{1, n, p^{\prime}}^{1}\left(\omega^{\prime}, z\right)\right\rangle=0$. However, although the expectation value of $\hat{X}_{1, n, p^{\prime}}\left(\omega^{\prime}, z\right)$ is zero, its variance is not due to the quantum-mechanical zero-point motion of excitons. In HRS it is the exciton system, by means of its vacuum fluctuations, which probes itself and determines the optical decay of biexcitons. 
Since in HRS as well as in FWM the biexciton grating is driven by the coherent laser field, we can replace in equation (9) the biexciton operator by the $c$-number corresponding to its semiclassical expectation value. The semiclassical expectation value of the biexciton operator is obtained taking the expectation value of equation (12) and performing the semiclassical factorization: $\left\langle\hat{X}_{0 ; 1}^{(1)} \hat{E}^{(1)}\right\rangle \rightarrow\left\langle\hat{X}_{0 ; 1}^{(1)}\right\rangle\left\langle\hat{E}^{(1)}\right\rangle$. By this approximation we are assuming that the field fluctuations in the input laser beam can be neglected with respect to the strong field expectation value. We consider one input pump beam, driving the biexciton amplitude, of given direction and frequency $\omega_{I}$, with amplitude $\left\langle E_{p_{I}}^{+}(\omega, 0)\right\rangle=E_{p_{I}}\left(\omega_{I}\right) \delta\left(\omega-\omega_{I}\right)$. After replacing the biexciton operator by its expectation value, we obtain for the biexciton amplitude the following expression

$\hat{X}_{2 \alpha, 2 p_{I}}^{(2)}(\omega, z)=\frac{\delta\left(\omega-2 \omega_{I}\right)}{\omega_{2, \alpha}-\omega} \sum_{n_{1}, n_{2}} M_{n_{2}}\left\langle 2, \alpha\left|\hat{B}_{n_{2}}\right| 1, n_{1}\right\rangle \times \chi_{n_{1}}\left(\omega_{I}\right) \mathrm{e}^{2 \mathrm{i} k_{p_{I}} z} E_{p_{I}}\left(\omega_{I}\right) E_{p_{I}}\left(\omega_{I}\right)$.

Introducing equation (17) into equation (14), the nonlinear source term can be written as

$$
\hat{S}_{n, \boldsymbol{p}}^{(3)}(\omega, z)=\sum_{n^{\prime}} \hat{X}_{1, n^{\prime}, \boldsymbol{p}^{\prime}}^{\dagger}\left(\omega^{\prime}, z\right) \chi_{n, n^{\prime}}\left(\omega_{I}, \omega_{I}\right) \mathrm{e}^{2 \mathrm{i} k_{p_{I}}(\omega) z} E_{\boldsymbol{p}_{I}}^{2}\left(\omega_{I}\right)
$$

where $\omega^{\prime}=2 \omega_{I}-\omega, \boldsymbol{p}^{\prime}=2 \boldsymbol{p}_{I}-\boldsymbol{p}$ and with the susceptibility function given by

$$
\chi_{n, n^{\prime}}=\sum_{n_{1} n_{2}} \frac{M_{n_{1}} M_{n_{2}}\left(Y_{n, n^{\prime}}^{n_{1}, n_{2}}-W_{n, n^{\prime}}^{n_{1}, n_{2}}\left(2 \omega_{I}\right)\right)}{\left(\omega_{1, n_{1}}-\omega_{I}\right)\left(\omega_{1, n_{2}}-\omega_{I}\right)} .
$$

In equation (19) $W\left(2 \omega_{I}\right)$ is the spectral distribution of the two-exciton correlation function defined and computed by a first principles numerical calculation in [10]. It contains resonances due to bound biexciton molecules as well as contributions from the continuum of unbound biexciton states. $Y$ describes exciton-exciton interactions as in the usual HFSBE. It is a first order term in the Coulomb interaction and, in contrast to $W$, it does not depend on biexciton states. These two terms read

$$
\begin{aligned}
& W_{\alpha \alpha^{\prime}}^{\alpha_{1} \alpha_{2}}\left(2 \omega_{I}\right)=\left\langle 0\left|D_{\alpha \alpha^{\prime}} \frac{1}{\hat{H}_{c}-2 \omega_{I}} D_{\alpha_{1} \alpha_{2}}^{\dagger}\right| 0\right\rangle \\
& Y_{\alpha \alpha^{\prime}}^{\alpha_{1} \alpha_{2}}=\left\langle 0\left|D_{\alpha \alpha^{\prime}} \hat{B}_{\alpha_{1}}^{\dagger} \hat{B}_{\alpha_{2}}^{\dagger}\right| 0\right\rangle
\end{aligned}
$$

where $\hat{D}_{\alpha \alpha^{\prime}}=\left[\hat{B}_{\alpha},\left[\hat{B}_{\alpha^{\prime}}, \hat{H}_{c}\right]\right]$. We also notice that, in principle, the phase space filling term in $\hat{R}^{(2)}$ contributes to the nonlinear source term giving rise to a further susceptibility term. However, for $2 \omega_{I}$ almost resonant with the bound biexciton energy level, this term and also the contribution from $Y$ are not important with respect to the correlation spectral distribution $W\left(2 \omega_{I}\right)$. Equation (20) has been obtained exploiting the fact that $\sum_{\alpha}|2, \alpha, \boldsymbol{k}\rangle\langle 2, \alpha, \boldsymbol{k}|$ is the unity operator for the biexciton subspace of given wave vector $k$. Now the lowest order source term is completely determined and is composed by a $c$-number which multiplies the operator $\hat{X}_{1, n^{\prime}, p^{\prime}}^{(1)}\left(\omega^{\prime}, z\right)$, whose equation of motion has been given in equation (11).

Once we have determined the source term for the scattering process, we can write down the equation of motion for the nonlinear polarization in the frequency domain, from which we obtain

$$
\hat{P}_{p}^{(3)}(\omega, z)=\sum_{n} \chi_{n}^{(1)}(\omega) \hat{S}_{n, p}^{(3)}(\omega, z)+M_{n}^{*} \chi_{n}^{(1)}(\omega) \hat{E}_{p}^{(3)}(\omega, z)
$$

In order to calculate the scattered field, as in classical nonlinear optics, we need to consider field propagation inside the medium. By including equation (21) and the linear polarization term

$$
\hat{P}_{p}^{(1)}(\omega, z)=M_{n}^{*} \chi_{n}^{(1)}(\omega) \hat{E}_{p}^{(1)}(\omega, z)
$$


into equation (13), we obtain the following wave equation

$$
\left(\frac{\partial^{2}}{\partial z^{2}}+k_{z}^{2}(\omega)\right) \hat{E}_{p}^{+}(\omega, z)=\frac{\omega^{2}}{\varepsilon_{0} c^{2}} \sum_{n} \chi_{n}^{(1)}(\omega) \hat{S}_{n, p}^{(3)}(\omega, z) .
$$

In equation (23) $k_{z}$ is the $z$ component of the wave vector inside the medium, satisfying the following polariton dispersion relation

$$
k^{2} \equiv p^{2}+k_{z}^{2}=\frac{\omega^{2}}{c^{2}} \varepsilon(\omega)
$$

with $\varepsilon(\omega)=\varepsilon_{b}+\sum_{n}\left|M_{n}\right|^{2} /\left[\varepsilon_{0}\left(\omega_{n}-\omega\right)\right]$.

Until now we have not taken into account dissipation. As is well known, excitons in semiconductors are an open quantum system in interactions with phonons and other dephasing mechanisms. We include attenuation due to the various scattering mechanisms acting on the polarization waves in a phenomenological way, by introducing the coupling of excitons with a large number of reservoir oscillators at temperature $T=0 \mathrm{~K}$. Following the usual Langevin approach, the resulting Heisenberg-Langevin equations for the electronic polarization include a damping term and a quantum-noise Langevin operator. As a consequence, when taking into account dephasing, $k_{z}=k_{z}^{\mathcal{R}}+\mathrm{i} k_{z}^{\mathcal{I}}$ becomes complex and describes waves attenuations inside the medium and the operator wave equation (23) include a noise-current term, which ensures preservation of commutation relations despite absorption. $k_{z}$ obeys equation (24) also in the presence of attenuation. In this case, however, the dielectric function $\varepsilon(\omega)$ is complex and it is given by $\varepsilon(\omega)=\varepsilon_{b}+\sum_{n}\left|M_{n}\right|^{2} /\left[\varepsilon_{0}\left(\omega_{n}-\omega-\mathrm{i} \gamma_{x}\right)\right]$. Correspondingly we also introduce a phenomenological dephasing rate $\Gamma_{2}$ for the semiclassical biexciton amplitude by replacing in equation (17) $\omega_{2, \alpha}$ with $\omega_{2, \alpha}-\mathrm{i} \Gamma_{2}$.

We consider a semiconductor slab of thickness $L$ with ideal anti-reflection coatings on its sides so that, for the range of frequency which we will take into account, the photon flux remains unaffected when crossing the interface. In vacuum, the field components $\hat{E}_{p}^{+}(z, \omega)$ can be written in terms of photon operators $\hat{E}_{p}^{+}(z, \omega)=K_{p}^{0}(\omega) \mathrm{e}^{\mathrm{i} k_{z}^{0} z} \hat{a}_{p}(\omega)$, where $k^{0}=\omega / c$ and $K_{p}^{0}(\omega)$ is a normalization coefficient ensuring satisfaction of the commutation rules for the light field. For future use it is useful to introduce the photon number operator as $\hat{n}_{p}(\omega)=\int_{\omega-\delta}^{\omega+\delta} \mathrm{d} \omega^{\prime} \hat{a}_{p}^{\dagger}\left(\omega^{\prime}\right) \hat{a}_{p}\left(\omega^{\prime}\right)$, where $2 \delta(>0$ and small) is the bandwidth of the photoncounter.

Applying the usual slowly varying envelope approximation, and integrating the wave equation equation (23), after a distance $z=L$ the electric-field operator is given by

$$
\hat{E}_{p}^{+}(\omega, L)=\hat{E}_{p}^{(1)}(\omega, L)+\hat{E}_{p}^{N L}(\omega, L)
$$

with the scattered field given by

$$
\begin{aligned}
\hat{E}_{p}^{N L}(\omega, L)= & \frac{\omega^{2}}{\varepsilon_{0} c^{2} 2 k_{z}} \sum_{n, n^{\prime}} \chi_{n}^{(1)}(\omega) \chi_{n, n^{\prime}}\left(\omega_{I}, \omega_{I}\right) E_{p_{I}}^{2}\left(\omega_{I}\right) \\
& \times \mathrm{e}^{\mathrm{i} k_{z} L} \int_{0}^{L} \mathrm{~d} z^{\prime} \mathrm{e}^{\mathrm{i}\left(2 k_{z I}-k_{z}\right) z^{\prime}} \hat{X}_{1, n^{\prime}, p^{\prime}}^{(1) \dagger}\left(\omega^{\prime}, z^{\prime}\right)
\end{aligned}
$$

where $\omega^{\prime}=2 \omega_{I}-\omega$ and $\boldsymbol{p}^{\prime}=2 \boldsymbol{p}_{I}-\boldsymbol{p}$. The scattered electric-field operator equation (26) is our starting-point for analysing coherent nonlinear processes related to the third order nonlinear susceptibility induced by a pump beam.

We first observe that, in the presence of a coherent probe light field $E_{p^{\prime}}\left(\omega^{\prime}\right)$, the exciton amplitude $\left\langle\hat{X}_{1, n^{\prime}, p^{\prime}}^{\dagger}\left(\omega^{\prime}, z^{\prime}\right)\right\rangle$ is driven according to equation (15), furthermore the expectation value of the operator $\hat{E}_{\boldsymbol{p}}^{(1)}(\omega, L)$ in equation (25) is zero as the light mode at $\boldsymbol{p} \neq \boldsymbol{p}_{I}, \boldsymbol{p}^{\prime}$ is not 
driven by any input light beam and the photon state in the Heisenberg picture contains zero photons of in-plane wave vector $\boldsymbol{p}$. Taking the expectation value of equation (26) we recover the semiclassical result describing FWM via biexcitons.

In HRS there is no input light beam at energy $\omega^{\prime}$ and in direction $\boldsymbol{p}^{\prime}$, the light mode at $\boldsymbol{p}^{\prime}$ is thus in the vacuum state and we obtain for the electric field in equation (25) $\left\langle\hat{E}_{\sigma p}^{(+)}\right\rangle=0$. Instead the steady state power emission spectrum, defined by

$$
I_{\sigma, p}\left(\omega_{s}, L\right) \propto \int \mathrm{d} \tau \mathrm{e}^{\mathrm{i} \omega_{s} \tau}\left\langle\hat{E}_{\sigma p}^{(-)}(t, z) \hat{E}_{\sigma p}^{(+)}(t+\tau, z)\right\rangle \propto \hat{n}_{p}\left(\omega_{s}\right)
$$

is different from zero. $I_{\sigma, p}\left(\omega_{s}, z\right) \mathrm{d} \omega_{s}$ is the intensity of light measured by an ideal narrowband photodetector able to select a narrow frequency band $\mathrm{d} \omega_{s}$ around $\omega_{s}$, and able to select a single propagation direction. In particular the detector selects a single mode propagating in the direction determined by the angle $\theta=\arcsin \left[c^{2} p^{2} / \omega^{2}\right]$ between the propagation direction and the $z$ axis, and by the azimuthal angle $\phi=\arctan \left(p_{y} / p_{x}\right)$.

In the following we analyse the nonlinear scattering process involving the lower polariton branch. We consider a monochromatic input light beam with frequency below the $1 \mathrm{~s}$ exciton level, which propagates inside the semiconductor as a polariton in the lower branch. We consider the case in which two photons of the input beam create a virtual biexciton state which spontaneously decays into two polaritons in the lower polariton branch. We label the final polariton quanta as 1 and 2. Energy and in-plane momentum are conserved, respectively $\omega_{1}+\omega_{2}=2 \omega_{I}$ and $\boldsymbol{p}_{1}+\boldsymbol{p}_{2}=\boldsymbol{p}_{I}$. By using the electric field operator given in equation (25), we can calculate the mean number of emitted photons at mode 1 and energy $\omega_{1}$, which is given by

$$
\left\langle\hat{n}_{1}\right\rangle=Q_{1,2}^{2}\left|\chi^{(3)} E_{I}^{2}\left(\omega_{I}\right)\right|^{2} g_{1}(L)
$$

where $Q_{1,2}=\omega_{1} \omega_{2} /\left(2 \varepsilon_{0} c^{2} \sqrt{k_{z_{1}}^{\mathcal{R}} k_{z_{2}}^{\mathcal{R}}}\right)$, and $g_{1}(z)$ describes the propagation and the phasematching resonance condition. It is given by

$g_{1}(z)=\frac{k_{z_{2}}^{\mathcal{R}}}{k_{z_{2}}}\left\{\frac{\mathrm{e}^{-2 k_{1}^{\mathcal{I}} z}}{2 k_{z_{I}}-k_{z_{1}}-k_{z_{2}}}\left(\frac{1-\mathrm{e}^{-2\left(2 k_{z_{I}}^{\mathcal{I}}-k_{z_{1}}^{\mathcal{I}}\right) z}}{2\left(2 k_{z_{I}}^{\mathcal{I}}-k_{z_{1}}^{\mathcal{I}}\right)}+\mathrm{i} \frac{1-\mathrm{e}^{-\mathrm{i}\left(\Delta k_{z}^{*}\right) z}}{\Delta k_{z}^{*}}\right)+\right.$ c.c. $\}$.

The susceptibility determining the third order nonlinear response is given by $\chi^{(3)}=$ $\sum_{n, n^{\prime}} \chi_{n^{\prime}}^{(1) *} \chi_{n, n^{\prime}} \chi_{n}^{(1)}$. The role of modes 1 and 2 can be interchanged. Thus the spontaneous decay of virtually excited biexcitons can give rise to two light beams 1 and 2 . They are sons of the virtually excited states with two eh pairs. Of course $\left\langle\hat{n}_{2}\right\rangle$ can be obtained from equation (28) simply by exchanging the labels 1 and 2 . The intensity of the scattered fields $\left\langle\hat{n}_{1,2}\right\rangle$, calculated at the phase-matching resonance condition $\left(\mathcal{R} e\left[\Delta k_{z}\right]=0\right)$, are displayed in figure 1 as a function of the incident energy $\omega_{I}$ for a $\mathrm{CuCl}$ slab of $50 \mu \mathrm{m}$. We have considered a forward scattering geometry with incident light orthogonal to the slab and observation angles $\simeq 10^{\circ}$. The two-exciton correlation $W\left(2 \omega_{I}\right)$ in the resonant nonlinear susceptibility was calculated by assuming one bound biexciton level with energy $\omega_{2}=6.372 \mathrm{eV}$ and with homogeneous broadening $\Gamma_{2}=1.6 \mathrm{meV}$. The exact two-exciton correlation $W\left(2 \omega_{I}\right)$ has been calculated numerically for a one-dimensional semiconductor model with long-range Coulomb interaction [10]. The various scattering mechanisms determine an increasing trend in the dephasing rate $\gamma$ as the energy approaches the exciton resonance. We have used a dephasing rate, displayed in the inset in figure 1, fitting quite well the experimental data [27]. The peak at higher energy $\left(\left\langle\hat{n}_{2}\right\rangle\right)$ is slightly lower than the other due to a larger degree of dephasing during propagation. The results shown in this section demonstrate the intimate connection of HRS with electromagnetic field quantization. The underlying microscopic scattering mechanisms of HRS involve many-body correlations and quantum-mechanical zero-point motion in a strong 


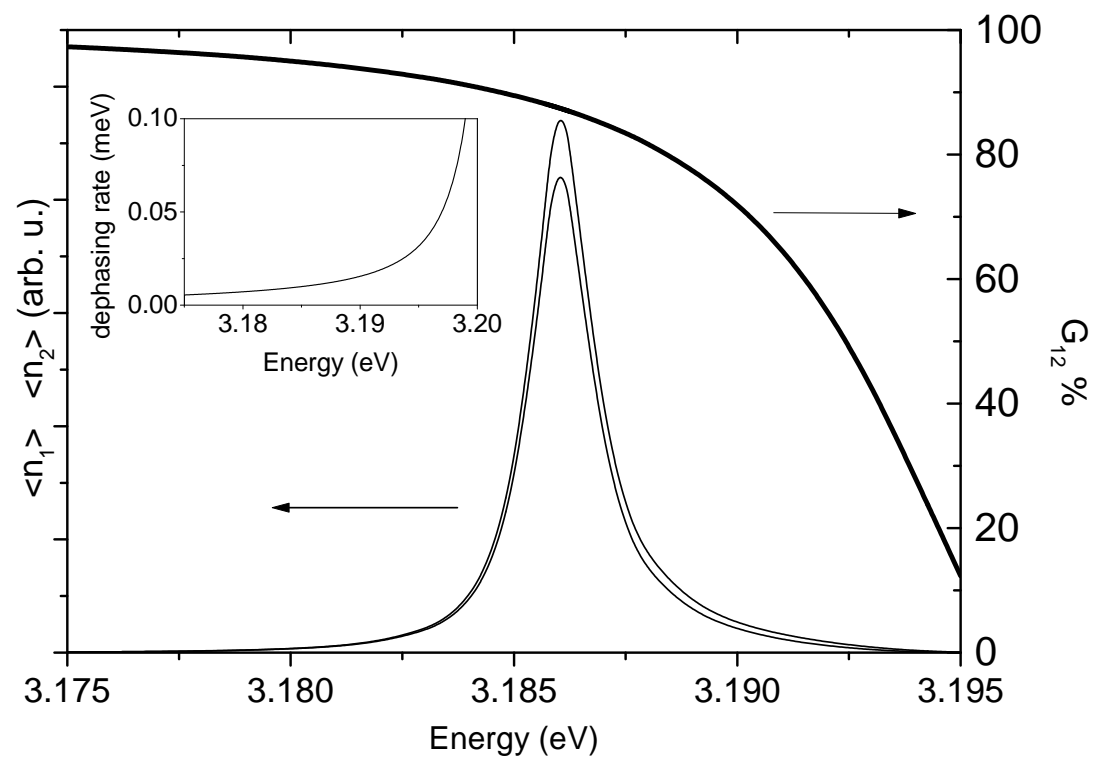

Figure 1. The mean number of emitted photons $\left\langle\hat{n}_{1,2}\right\rangle$ and the degree of entanglement $\mathcal{G}_{12}$ calculated at the phase-matching resonance condition as a function of the incident energy for a $\mathrm{CuCl}$ slab of thickness $L=50 \mu \mathrm{m}$. The inset shows the dephasing rate as a function of energy used for the calculations.

coupling regime. The results shown here indicate that HRS has to be regarded not only as an efficient technique for the investigation of polariton dispersion but also as a novel promising tool to explore excitonic quantum dynamics as we wiil see in the next section.

\section{Entangled photon pairs from the optical decay of biexcitons}

The electric field operator for third order nonlinear processes calculated in the previous section allows us to also calculate higher order field-field correlations. In the following we analyse the correlation properties of the light emitted in the HRS process. We present results for a $\mathrm{CuCl}$ slab and for III-V semiconductor microcavities.

\subsection{Slab of $\mathrm{CuCl}$}

We first consider the two-mode intensity correlation functions $\left\langle\hat{n}_{2} \hat{n}_{1}\right\rangle$. By using the electric field operator given in equation (25), we obtain

$$
\left\langle\hat{n}_{1} \hat{n}_{2}\right\rangle=\left\langle\hat{n}_{2} \hat{n}_{1}\right\rangle=Q_{1,2}^{2}\left|\chi^{(3)} E_{I}^{2}\left(\omega_{I}\right)\right|^{2} g_{1,2}(L)
$$

where

$$
g_{1,2}(z)=\mathrm{e}^{-2\left(k_{z_{1}}^{T}+k_{z_{2}}^{T}\right) z}\left|\frac{1-\mathrm{e}^{\left(2 k_{z_{1}}-k_{z_{1}}-k_{z_{2}}\right) z}}{2 k_{z_{I}}-k_{z_{1}}-k_{z_{2}}}\right|^{2} .
$$

As we have already observed, modes 1 and 2 are not independent but are related by total energy and momentum conservation. For each other mode $m$, different from mode 2 and from the input mode $I$, we obtain (in the low density limit) $\left\langle: \hat{n}_{1} \hat{n}_{m}:\right\rangle=0$ (:: denotes normal order). This is due to the fact that, at lowest order, photons are created in pairs and, if 1 and $m$ do not belong to the same pair, $\left\langle: \hat{n}_{1} \hat{n}_{m}:\right\rangle \neq 0$ implies a higher order scattering process. As a 
consequence, the single-mode correlation functions, calculated in the low density limit, are zero. In particular we obtain $\left\langle: \hat{n}_{1}^{2}:\right\rangle=\mathcal{O}\left(E_{I}^{6}\right)$, where $\mathcal{O}\left(E_{I}^{6}\right)$ indicates those terms of order $\geqslant 6$.

The intensity correlation functions in the low density limit exhibit quantum features as they violate the classical Cauchy-Schwartz inequality, $\left\langle: \hat{n}_{2} \hat{n}_{1}:\right\rangle^{2} \leqslant\left\langle: \hat{n}_{1}^{2}:\right\rangle\left\langle: \hat{n}_{2}^{2}:\right\rangle$. Entangled photon pairs exhibit fourth order interference in the joint detection probability that cannot be obtained with classical systems [18]. In classical systems the visibility of interference fringes cannot exceed 50 per cent. The visibility of interference can be written in terms of the fourth order correlations as $U=2\left\langle: \hat{n}_{2} \hat{n}_{1}:\right\rangle /\left(\left\langle: \hat{n}_{1}^{2}:\right\rangle+\left\langle: \hat{n}_{2}^{2}:\right\rangle+2\left\langle: \hat{n}_{2} \hat{n}_{1}:\right\rangle\right)$. By using the correlations calculated above we obtain $U=1-\mathcal{O}\left(E_{I}^{6}\right)$, i.e. fringe visibility reaching 100 per cent in the low density limit. Thus fourth order interference could be used to observe experimentally the quantum features of light emerging from the spontaneous decay of biexcitons.

We can quantify the degree of quantum entanglement, by using the ratio

$$
\mathcal{G}_{1,2} \equiv\left\langle: \hat{n}_{2} \hat{n}_{1}:\right\rangle / \sqrt{\left\langle\hat{n}_{1}^{2}\right\rangle\left\langle\hat{n}_{2}^{2}\right\rangle} \leqslant 1
$$

which is amaximum for ideal entangled pairs. By using the correlation functions calculated above, in the low density limit, we obtain

$$
\mathcal{G}_{1,2}=\frac{g_{12}(L)}{\sqrt{g_{1}(L) g_{2}(L)}} .
$$

In the absence of attenuation, $g_{12}(L)=g_{1}(L)=g_{2}(L)$ and we obtain $\mathcal{G}_{1,2}=1$. Thus we can conclude that the optical decay of states with two electron-hole pairs, for negligible absorption, produces an ideal entangled pair of photons. Of course photon reabsorption tends to destroy ideal entanglement. In particular the degree of entanglement is affected by those events which, after the biexciton decay, scatter one polariton of the pair. A simple criterium for negligible reabsorption is $k^{\mathcal{I}} L \ll 1$. Thus the degree of entanglement depends strongly on the energy of the scattered light and on the length of the slab. Figure 1 displays $\mathcal{G}_{1,2}$ as a function of the incident energy. Reabsorption causes $\mathcal{G}_{1,2}$ to go rapidly to zero as $\Omega_{I}$ approaches the energy of the $1 \mathrm{~s}$ exciton level $\omega_{1}=3.2026 \mathrm{eV}$. The noticeable biexciton binding energy, determined by the Coulomb interaction between excitons, in $\mathrm{CuCl}$ (as well as in other large gap semiconductors) permits the detection of hyper Raman lines by using incident light with energy sufficiently far from the exciton level to prevent strong reabsorption. This permits the polariton pairs to escape the crystal with a high degree of entanglement, as shown in figure 1. The pair formation and reabsorption during propagation is shown in figure 2, which gives information on the sample thickness more convenient to observe the photon pairs.

\subsection{Cavity polaritons}

With the development of crystal growth technologies it has become possible to realize semiconductor systems where both electrons and photons are confined along one or more directions. When a quantum well $(\mathrm{QW})$ is placed inside a planar $\mathrm{MC}$, dramatic changes in the optical response can be produced [23]. In contrast to bulk materials, the optical properties can be tailored by the design of the cavity and of the embedded QWs. In the following we will briefly show how device's design affects the entanglement of polariton pairs. We treat the cavity field in the quasi-mode approximation, the cavity field is quantized as though the mirrors where perfect, and the resulting modes, whose annihilation operators in the Heisenberg representation are labelled by $\hat{a}_{p}(t)$, are coupled by an interaction term to the external continuum of modes. The linear coupling of cavity modes with the external modes provides both the damping and the input optical pumping of the cavity modes. We have applied this approximation 


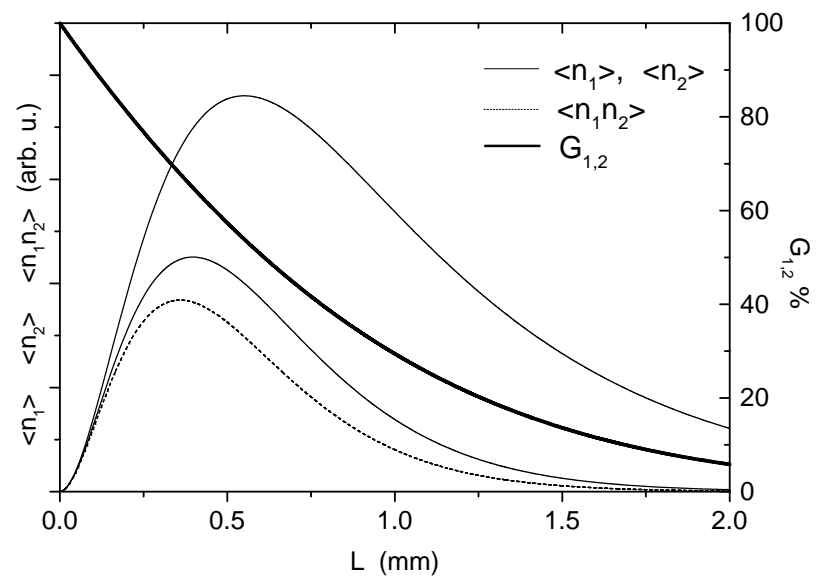

Figure 2. The mean number of emitted photons $\left\langle\hat{n}_{1,2}\right\rangle$, the pair correlations $\left\langle\hat{n}_{1} \hat{n}_{2}\right\rangle$, and the degree of entanglement $\mathcal{G}_{12}$ calculated at the phase-matching resonance condition as a function of the slab thickness.

scheme to analyse quantum optical effects in cavity embedded electron systems $[14,15]$. We have calculated the normalized power spectrum of noise in the intensity difference of the two output beams emerging from HRS, following the analogous Heisenberg-Langevin perturbative treatment that we used for the bulk case. The pertinent Heisenberg-Langevin equations for the exciton-photon system can be found in [15].

As for the bulk case we label the final polariton quanta as 1 and 2 . In order to test the nonclassical behaviour of the emitted photon pairs, we calculate the normalized spectrum of fluctuations in the output intensity difference $\hat{I}_{1}-\hat{I}_{2}$, where $\hat{I}_{1(2)}=2 \gamma_{c} \hat{a}_{1(2)}^{\dagger}(t) \hat{a}_{1(2)}(t)$. The normalized spectrum of fluctuations is defined by

$$
S(\Omega)=\frac{\int \mathrm{d} \tau \mathrm{e}^{-\mathrm{i} \Omega \tau}\left\langle\Delta\left(\hat{I}_{1}(\tau)-\hat{I}_{2}(\tau)\right) \Delta\left(\hat{I}_{1}(0)-\hat{I}_{2}(0)\right)\right\rangle}{2 \gamma_{c}\left(\left\langle\hat{I}_{1}(0)\right\rangle+\left\langle\hat{I}_{2}(0)\right\rangle\right)}
$$

where for a generic operator $\hat{x}, \Delta \hat{x}=\hat{x}-\langle\hat{x}\rangle$. We find that the intensity difference carries fluctuations below the shot noise level $(S(\Omega)<1)$. The normalized power spectrum of noise in the intensity difference field $S(\Omega)>0$ cannot be less than 1 in classical fields. Furthermore, ideal entangled states displays at $\Omega=0$ complete noise reduction $(\mathrm{S}(0)=0)$. We do not report here the resulting expressions for $S(\Omega)$ as they are somewhat lengthy and contains numerical integrations. In figure 3 we display the percent noise reduction $1-S(0)$ as a function of the photon escape-rate through the MC's mirrors $\gamma_{c}$, calculated for two different values of the exciton dephasing rate $\gamma_{x}$. We have chosen the pump energy in between the Rabi peaks and at normal incidence as shown in figure 4. The cavity is resonant with the bare exciton energy. We have used $V=5.6 \mathrm{meV}$ for the exciton-photon coupling. Figure 3 clearly shows that, in contrast to bulk systems, an important level of entanglement can be produced, also at resonance and taking into account realistic exciton dephasing rates and exciton-photon couplings for III$\mathrm{V}$ semiconductor MCs. The results displayed in figure 3 can be explained observing that in MCs at zero detuning, the criterion for small reabsorption is $\gamma_{x} \ll \gamma_{c}$. The condition $\gamma_{x} \ll \gamma_{c}$ implies that the photon pairs can escape the MC before being reabsorbed or scattered by the exciton dephasing. However as shown in figure 3, the noise suppression for $\gamma_{x} \ll \gamma_{c}$ is not $100 \times 100$ and depends on $\gamma_{x}$. This can be understood by observing that the photon pairs are emitted from the QW, and also for high values of $\gamma_{c}$ they are expected to feel the presence of 


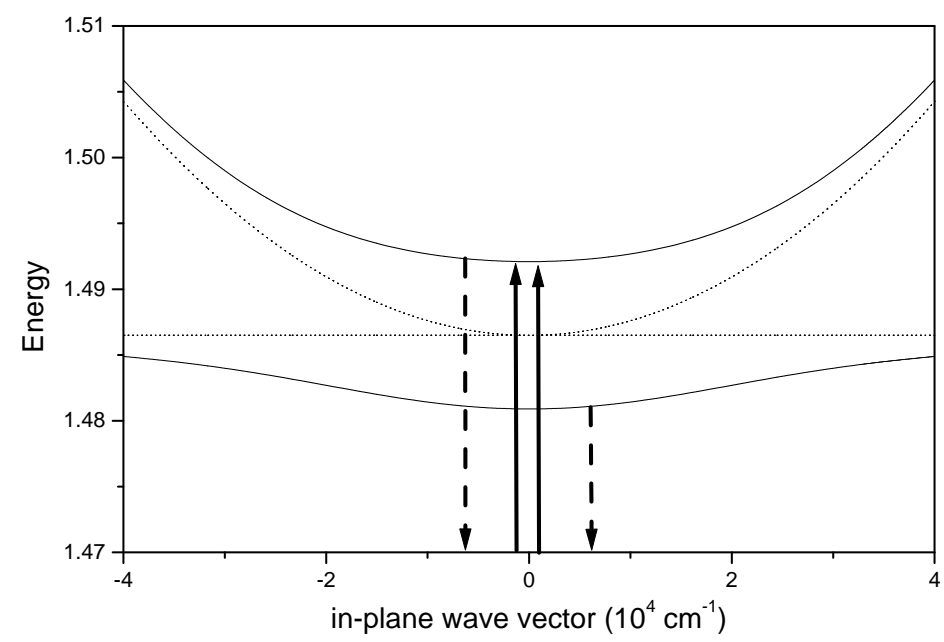

Figure 3. Percentage noise reduction in the intensity difference between the emitted output photon pairs $1-S(0)$ in a resonantly excited semiconductor microcavity as a function of the photon escape rate $\gamma_{c}$. The inset dysplays the normalized noise spectrum $S(\omega)$. The parameters are given in the text.

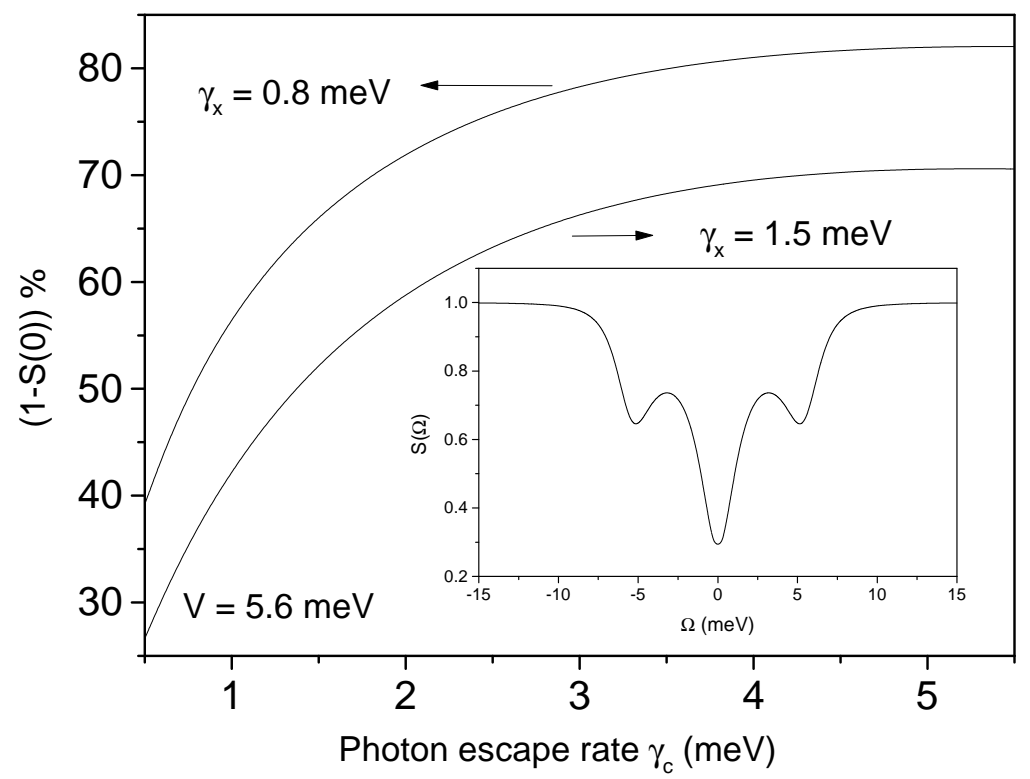

Figure 4. Schematic description of the resonant hyper Raman scattering process in a semiconductor microcavity. The continuous arrows represent the pump photons, while the dashed arrows represent the emitted photons.

the absorbing QW.

Even higher levels of entanglement can be obtained by detuning the MCs from the exciton energy and tuning the energy of the input light beam so that energy conservation implies pairs of photon-like polaritons emerging from the scattering process. The inset in figure 3 shows one normalized noise spectrum calculated by using $\gamma_{x}=0.8 \mathrm{meV}$ and $\gamma_{c}=2 \mathrm{meV}$. 


\section{Conclusion}

In this paper we have presented a fully quantum mechanical theory for an interacting system of photons and Coulomb correlated electrons and holes in semiconductors using a pertubation series in the exciting laser field. We have applied the theory to analyse the signatures of the electromagnetic field quantization in the third order onlinear optical response of excitons. We have shown that it is possible to transfer the exciton-exciton Coulomb correlation to photons, producing thus pairs of near-gap photons with a high degree of quantum correlation.

\section{References}

[1] Allen L and Eberly J H 1975 Optical Resonance and Two-Level Atoms (New York: Wiley)

[2] Meystre P and Sargent M III 1990 Elements of Quantum Optics 2nd edn (Berlin: Springer)

[3] See, e.g., Haugh H and Koch S W 1993 Quantum Theory of the Optical and Electronic Properties of Semiconductors 2nd edn (Singapore: World Scientific)

[4] Lindberg M and Koch S W 1988 Phys. Rev. B 383342

[5] Schmitt-Rink S and Chemla D S 1986 Phys. Rev. Lett. 572752

[6] Chemla D S, Bigot J-Y, Mycek M-A, Weiss S and Schäfer W 1994 Phys. Rev. B 508439

[7] Combescot M and Combescot R 1988 Phys. Rev. Lett. 61117

Combescot M and Combescot R 1989 Phys. Rev. B 403788

[8] Axt V M and Sthal K 1994 Z. Phys. B 93205

[9] Victor K, Axt V M and Stahl A 1995Phys. Rev. B 5114164

[10] Östreich Th, Schönhammer K and Sham L J 1995 Phys. Rev. Lett. 744698

[11] Kira M et al 1997 Phys. Rev. Lett. 79 5170,

[12] For a review, see Hönerlage B, Levy R, Grun J B, Klingshirn C and Bohnert K 1985 Phys. Rep. 124161

[13] See, e.g., Scully M O and Zubairy M S 1997 Quantum Optics (Cambridge: Cambridge University Press)

[14] Savasta S and Girlanda R 1996 Phys. Rev. Lett. 774736

[15] Savasta S, Girlanda R and Martino G 1997 Phys. Status Solidi a 16485

[16] Savasta S and Girlanda R 1999 Phys. Rev. B at press

[17] Greenberger D M et al 1993 Phys. Today 46 no 8, 22 and references therein

[18] Gosh R and Mandel L 1987 Phys. Rev. Lett. 591903

[19] Keller C et al 1997 Nature 390575

[20] Boschi D et al 1998 Phys. Rev. Lett. 801121

[21] Di Vincenzo D P 1995 Science 270255

[22] Bennett C H 1992 Phys. Rev. Lett. 683121

[23] Weisbuch C et al 1992 Phys. Rev. Lett. 693314

[24] Östreich Th and Schönhammer K 1993 Solid State Commun. 85629

[25] Ivanov A L and Haug H 1993 Phys. Rev. B 481490

[26] Axt V M, Victor K and Stahl A 1996 Phys. Rev. B 537244

[27] Masumoto Y, Shionoya S and Takagahara T 1983 Phys. Rev. Lett. 51923 